\title{
Geometry and kinematics for a spherical-base integrated parallel mechanism
}

\author{
Jie Sun · Xinsheng Zhang • Guowu Wei • \\ Jian S. Dai (i)
}

Received: 27 October 2014/Accepted: 2 March 2016/Published online: 4 May 2016

(C) The Author(s) 2016. This article is published with open access at Springerlink.com

\begin{abstract}
Parallel mechanisms, in general, have a rigid base and a moving platform connected by several limbs. For achieving higher mobility and dexterity, more degrees of freedom are introduced to the limbs. However, very few researchers focus on changing the design of the rigid base and making it foldable and reconfigurable to improve the performance of the mechanism. Inspired by manipulating an object with a metamorphic robotic hand, this paper presents for the first time a parallel mechanism with a reconfigurable base. This novel spherical-base integrated parallel mechanism has an enlarged workspace compared with traditional parallel manipulators. Evolution and structure of the proposed parallel mechanism is introduced and the geometric constraint of the mechanism is investigated based on mechanism decomposition. Further, kinematics of the proposed mechanism is reduced to the solution of a univariate polynomial of degree 8 . Moreover, screw theory based Jacobian is presented followed by the velocity analysis of the mechanism.
\end{abstract}

J. Sun · X. Zhang · J. S. Dai ( $₫)$

Centre for Robotics Research, King's College London, University of London, Strand, London WC2R 2LS, UK e-mail: jian.dai@kcl.ac.uk

G. Wei

School of Computing, Science and Engineering, University of Salford, Salford, Manchester M5 4WT, UK
Keywords Parallel mechanism $\cdot$ Reconfigurable base $\cdot$ Metamorphic hand $\cdot$ Kinematics $\cdot$ Screw theory $\cdot$ Jacobian

\section{Introduction}

A typical parallel mechanism consists of a moving platform that is connected to a fixed base by several (at least two) limbs or legs. In general, the moving platform of parallel mechanisms has both rotational and translational motion [1, 2]. However, in order to reduce the complexity and cater some specific applications, the low-mobility parallel mechanisms [3-6] have drawn numerous interests from researchers in mechanism and machine design. In particular, Chablat and Wenger [7] proposed a 3-DOF parallel mechanism to realise three axes rapid machining applications. Zhao et al. $[8,9]$ investigated three and four DOFs parallel mechanisms relying on equivalent screw groups. Kong and Gosselin [10] presented several parallel mechanisms relying on screw theory based type synthesis method. Similarly, $\mathrm{Xu}$ and $\mathrm{Li}$ [11] applied screw theory to analyse the mobility and stiffness of an over-constrained 3-PRC parallel mechanism and converted it into a non-over-constrained 3-CTC parallel mechanism of the same mobility and kinematic properties. Huda and Takeda [12] invented a 3-URU parallel mechanism with three dimensional rotations. Such parallel mechanisms were widely adopted to achieve wrist-like motion, such as Argos, 
proposed by Vischer and Clavel [13] and the 3-RUU mechanism, proposed by Gregorio [14]. Gan and Dai [15] studied constraint screw systems of a 3-PUP parallel mechanism and revealed the influence between them and limb arrangements. Zhang et al. [16] discussed the constraint singularity and analysed the bifurcated motion of a 3-PUP parallel mechanism and the conversion between two bifurcated motion branches. In addition, some redundant parallel mechanisms [17, 18] were put forward to avoid singularities and obtain better kinematic properties.

The parallel mechanism mentioned above are all composed of rigid base and non-reconfigurable moving platform. In other words, their base or moving platform is a component with zero DOF rather than a mechanism with additional moving capability. Recently, the parallel mechanisms with reconfigurable features have been capturing attentions from the researchers in the fields of mechanisms and robotics. Based on the concept reconfigurability and principle of metamorphosis [19], Gan et al. [20] proposed a reconfigurable Hooke (rT) joint and presented a new metamorphic parallel mechanism that was capable of changing mobility and topological configurations. Zhang et al. [21] identified an axis-variable (vA) joint based on origami fold [22] leading to the development of a metamorphic parallel mechanism that had the capability of changing its mobility from 3 to 6 DOF. Wei and Dai [23] proposed a variable revolute (vR) joint with application to the constructure of a family of reconfigurable and deployable Platonic mechsnisms.

In addition, there is another kind of metamorphic parallel mechanisms that can reconfigure themselves through changing the configurations of their moving platform. Yi et al. [24] presented a flexible folded parallel gripper to meet the requests of both grasping and positioning objects with irregular shape and size. Mohamed and Gosselin [25] presented a kind of parallel mechanisms with reconfigurable platforms and analysed redundancy of proposed parallel mechanisms. Lambert [26] presented and analysed mobility and kinematics of a PentaG robot, which is a parallel mechanism with a flexible planar platform providing 5 DOFs in total.

In contrast to the above flexible-platform parallel mechanisms, the concept of parallel mechanisms with a foldable/reconfigurable base can be brought up but no literature shows the relevant investigation. Inspired by the grasp and manipulation of an object with a metamorphic hand containing a reconfigurable palm
(Fig. 1) [27-31], in this paper, a parallel mechanism with a reconfigurable base is for the first time proposed. The base of this parallel mechanism is formed by a spherical five-bar linkage, which provides augmented motion for each limb. Structure design of the proposed spherical-base integrated parallel mechanism is introduced, and geometry and kinematics of the mechanism are investigated leading to closed-form solutions. Screw theory [32] based Jacobian is then presented followed by the velocity analysis.

\section{A spherical-base integrated parallel mechanism}

2.1 From manipulation with a metamorphic hand to a parallel mechanism with a reconfigurable base

Figure 1 illustrates a three-fingered metamorphic robotic hand grasping and manipulating a disk. The metamorphic robotic hand (Metahand) contains a reconfigurable palm and three two-phalanx fingers. The reconfigurable palm is formed by a spherical fivebar linkage, with link AE as a grounded link, and the other four links are symmetrically arranged with respect to link $\mathrm{AE}$ such that links $\mathrm{AB}$ and $\mathrm{ED}$ are of the same length and so do links BC and DC. The three fingers are respectively mounted on link $\mathrm{AE}$ at point $A_{1}$, on link $D C$ at point $A_{2}$ and on link $B C$ at point $A_{3}$. When the palm is in a configuration that all the links are in a same plane, the three points $\mathrm{A}_{1}, \mathrm{~A}_{2}$ and $\mathrm{A}_{3}$ are

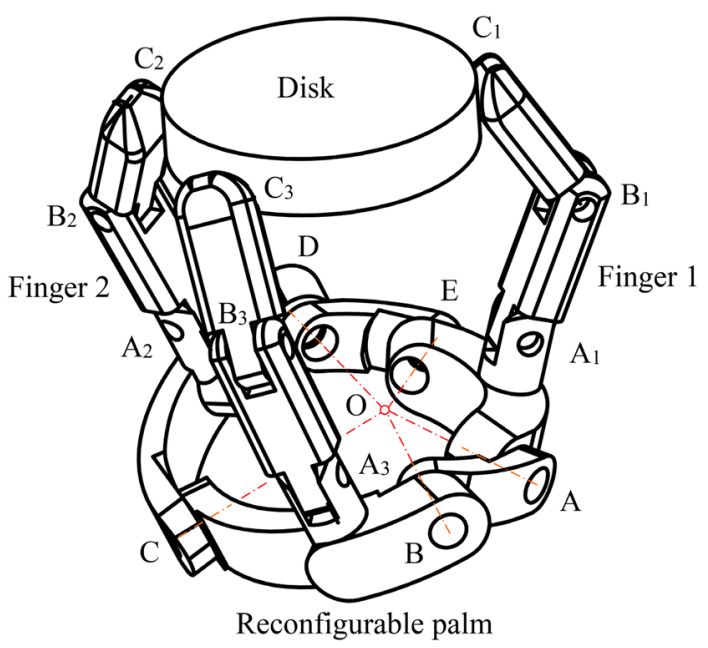

Fig. 1 Object manipulated by a three-fingered metamorphic hand 
evenly distributed about point $\mathrm{O}$, i.e. centre of the spherical five-bar linkage. The 2-DOF reconfigurable palm changes configuration of the whole hand, and increases workspace, dexterity and manipulability of the hand $[30,31]$. The idea of equivalence about considering the multi-fingered hand with a grasped object as a parallel mechanism was put forward by Borras-Sol and Dollar [33, 34]. When the hand is used to grasp and manipulate an object such as a disk as shown in Fig. 1, if the contact points between the object and the fingertips are thought of as spherical joints, an equivalent parallel mechanism with a reconfigurable base can be intuitively generated as illustrated in Fig. 2. This parallel mechanism is coined in this paper spherical-base integrated parallel mechanism.

\subsection{Structure of the spherical-base integrated parallel mechanism}

As shown in Fig. 2, the spherical-base integrated parallel mechanism is composed of a spherical reconfigurable base, a moving platform and three identical revolute-revolute-spherical chain connecting them. The reconfigurable base consists of five links which connect to each other forming a spherical five-bar linkage. In this design, link $\mathrm{AE}$ is fixed and joints $\mathrm{A}$ and $\mathrm{E}$ are assumed to be active joints to change the

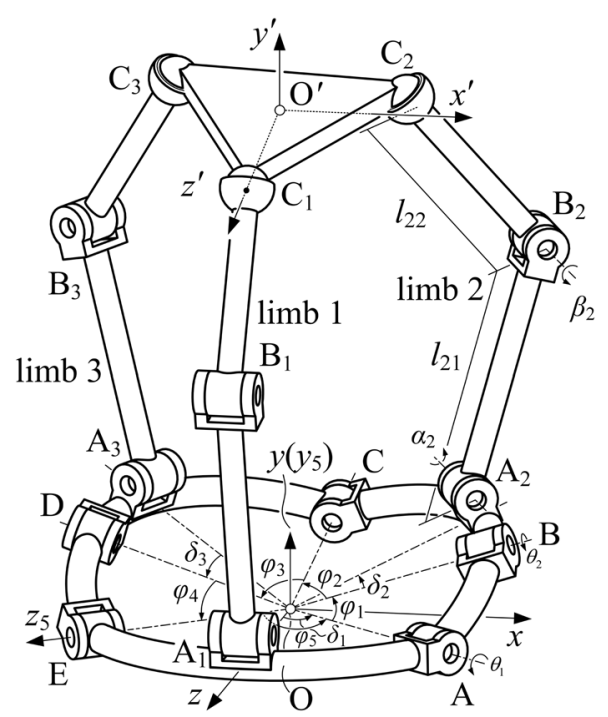

Fig. 2 Structure of the spherical-base integrated parallel mechanism configurations of the base, and joints $\mathrm{B}, \mathrm{C}$ and $\mathrm{D}$ are passive joints. The axes of these five joints always intersect at point $\mathrm{O}$. The angles covered by links $\mathrm{AB}$, $\mathrm{BC}, \mathrm{CD}, \mathrm{DE}$ and EA are denoted as $\varphi_{1}$ throughout $\varphi_{5}$ separately, and the sum of which satisfies $\varphi_{1}+\varphi_{2}+\varphi_{3}+\varphi_{4}+\varphi_{5}=2 \pi$. There are three identical limbs mounted at point $\mathrm{A}_{i}(i=1,2$ and 3), and the angles between $\mathrm{OA}_{1}$ and $\mathrm{OA}, \mathrm{OB}$ and $\mathrm{OA}_{2}$, $\mathrm{OA}_{3}$ and $\mathrm{OD}$ are indicated as $\delta_{1}, \delta_{2}$ and $\delta_{3}$. The angle between any two limbs is $120^{\circ}$ in the initial configuration of the mechanism when the five links of the reconfigurable base are located in a same horizontal plane. However, one has to clarify that this initial state of the mechanism as a singular configuration is suitable for theoretical computations rather than a starting configuration for practical applications. Each limb is made up of two linkages coupled by a revolute joint $\mathrm{B}_{i}(i=1,2$ and 3$)$. The limbs are connected to the reconfigurable base by revolute joints $\mathrm{A}_{i}(i=1,2$ and 3$)$ and the moving platform by spherical joints $C_{i}(i=1,2$ and 3). The length of link $\mathrm{A}_{i} \mathrm{~B}_{i}$ is denoted as $l_{i 1}$, while that of link $\mathrm{B}_{i} \mathrm{C}_{i}$ is denoted as $l_{i 2}(i=1,2$ and 3$)$.

\section{Mechanism decomposition and geometric constraints of the spherical-base integrated parallel mechanism}

The spherical-base integrated parallel mechanism, as the combination of a five-bar spherical base and a typical three-limb parallel mechanism, is a hybrid mechanism and it is complicated to analyse its kinematics directly. Therefore, using mechanism decomposition, analysis of the geometry constraint of the mechanism can be separated as that of the reconfigurable base and that of the normal parallel mechanism first and then combine them together.

\subsection{Constraint equations of the reconfigurable base}

As shown in Fig. 2, a global coordinate system F $\{\mathrm{O}-x y z\}$ is attached to the reconfigurable base with point $\mathrm{O}$ as the origin and its $y$-axis directed towards the upper platform and perpendicular to the plane formed by the axes of joints $\mathrm{A}$ and $\mathrm{E}$. The $z$-axis of the coordinate system lies along $\mathrm{OA}_{1}$. The radius of the spherical base is set at 1 for simplifying the calculation. For solving the geometric relationship of the reconfigurable base, 
local coordinate systems $\mathrm{M}_{i}\left\{\mathrm{O}-x_{i} y_{i} z_{i}(i=1\right.$ to 5$\left.)\right\}$ are created at point $\mathrm{O}$ with the $z_{i}$-axis aligned the joint axis (joint $\mathrm{A}, \mathrm{B}, \mathrm{C}, \mathrm{D}$ and $\mathrm{E}$ respectively) and the $y_{i}$-axis perpendicular to $z_{i} \times z_{i+1}$ (if $i=5, z_{i+1}$-axis represents $z_{1}$-axis). The coordinate system $\mathrm{M}_{5}\left\{\mathrm{O}-x_{5} y_{5} z_{5}\right\}$ is taken as an example indicated in Fig. 3. In this local coordinate system, the position vectors of point A, B, $\mathrm{C}, \mathrm{D}$ and $\mathrm{E}$ can be calculated as

$$
\boldsymbol{P}_{A}=\mathbf{R}\left(y_{5}, \varphi_{5}\right) \boldsymbol{P}_{E}=\left[\begin{array}{l}
s \varphi_{5} \\
0 \\
c \varphi_{5}
\end{array}\right]=\left[\begin{array}{l}
x_{A} \\
y_{A} \\
z_{A}
\end{array}\right], \quad \text { (1) } \quad \begin{array}{r}
\boldsymbol{P}_{\mathrm{C}}^{\mathrm{T}} \cdot \boldsymbol{P}_{\mathrm{D}}=c \varphi_{3}, \\
\boldsymbol{P}_{\mathrm{C}}^{\mathrm{T}} \cdot \boldsymbol{P}_{\mathrm{C}}=1 .
\end{array}
$$$$
\boldsymbol{P}_{\mathrm{B}}=\mathbf{R}\left(y_{5}, \varphi_{5}\right) \mathbf{R}\left(z_{1}, \theta_{1}\right) \mathbf{R}\left(y_{1}, \varphi_{1}\right) \boldsymbol{P}_{\mathrm{E}}
$$$$
=\left[\begin{array}{l}
c \varphi_{1} s \varphi_{5}+s \varphi_{1} c \varphi_{5} c \theta_{1} \\
s \varphi_{1} s \theta_{1} \\
c \varphi_{1} c \varphi_{5}-s \varphi_{1} s \varphi_{5} c \theta_{1}
\end{array}\right]=\left[\begin{array}{l}
x_{\mathrm{B}} \\
y_{\mathrm{B}} \\
z_{\mathrm{B}}
\end{array}\right],
$$$$
\boldsymbol{P}_{\mathrm{C}}=\mathbf{R}\left(z_{5},-\theta_{5}\right) \mathbf{R}\left(y_{4},-\varphi_{4}\right) \mathbf{R}\left(z_{4},-\theta_{4}\right) \mathbf{R}\left(y_{3},-\varphi_{3}\right) \boldsymbol{P}_{\mathrm{E}}
$$$$
=\left[\begin{array}{l}
s \varphi_{3}\left(s \theta_{4} s \theta_{5}-c \varphi_{4} c \theta_{4} c \theta_{5}\right)-c \varphi_{3} s \varphi_{4} c \theta_{5} \\
c \varphi_{3} s \varphi_{4} s \theta_{5}+s \varphi_{3}\left(s \theta_{4} c \theta_{5}+c \varphi_{4} c \theta_{4} s \theta_{5}\right) \\
c \varphi_{3} c \varphi_{4}-s \varphi_{3} s \varphi_{4} c \theta_{4}
\end{array}\right]
$$$$
=\left[\begin{array}{l}
x_{\mathrm{C}} \\
y_{\mathrm{C}} \\
z_{\mathrm{C}}
\end{array}\right]
$$

$$
\begin{aligned}
\boldsymbol{P}_{\mathrm{D}} & =\mathbf{R}\left(z_{5},-\theta_{5}\right) \mathbf{R}\left(y_{4},-\varphi_{4}\right) \boldsymbol{P}_{\mathrm{E}}=\left[\begin{array}{c}
-s \varphi_{4} c \theta_{5} \\
s \varphi_{4} s \theta_{5} \\
c \varphi_{4}
\end{array}\right] \\
& =\left[\begin{array}{c}
x_{\mathrm{D}} \\
y_{\mathrm{D}} \\
z_{\mathrm{D}}
\end{array}\right],
\end{aligned}
$$

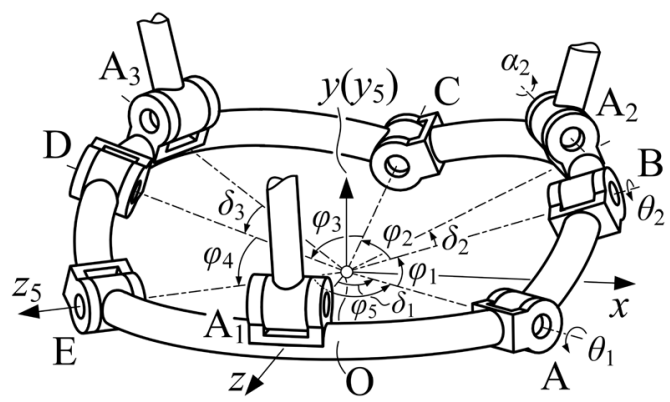

Fig. 3 The reconfigurable base of the spherical-base integrated parallel mechanism where $s$ and $c$ denote the sine and cosine functions, $\theta_{1}$ to $\theta_{5}$ are the rotation angles of joints $A$ to $E$, and $\boldsymbol{P}_{\mathrm{E}}=(0,0,1)^{\mathrm{T}}$.

Due to the geometric constraints of the spherical base, the position vectors of its joints have to satisfy the following equations,

$\boldsymbol{P}_{\mathrm{C}}^{\mathrm{T}} \cdot \boldsymbol{P}_{\mathrm{B}}=c \varphi_{2}$,

Substituting Eqs. (2), (3) and (4) into Eqs. (5) and (6) leads to the coordinates of $x_{\mathrm{C}}$ and $y_{\mathrm{C}}$ represented in terms of $z_{\mathrm{C}}$ as

$x_{\mathrm{C}}=P+Q z_{\mathrm{C}}$,

$y_{\mathrm{C}}=M+N z_{\mathrm{C}}$,

where

$$
\begin{gathered}
P=\frac{y_{\mathrm{D}} c \varphi_{2}-y_{\mathrm{B}} c \varphi_{3}}{x_{\mathrm{B}} y_{\mathrm{D}}-y_{\mathrm{B}} x_{\mathrm{D}}}, Q=\frac{y_{\mathrm{B}} z_{\mathrm{D}}-z_{\mathrm{B}} y_{\mathrm{D}}}{x_{\mathrm{B}} y_{\mathrm{D}}-y_{\mathrm{B}} x_{\mathrm{D}}}, \\
M=\frac{x_{\mathrm{B}} c \varphi_{3}-x_{\mathrm{D}} c \varphi_{2}}{x_{\mathrm{B}} y_{\mathrm{D}}-y_{\mathrm{B}} x_{D}}, N=\frac{z_{\mathrm{B}} x_{\mathrm{D}}-x_{\mathrm{B}} z_{\mathrm{D}}}{x_{\mathrm{B}} y_{\mathrm{D}}-y_{\mathrm{B}} x_{\mathrm{D}}} .
\end{gathered}
$$

Substituting Eqs. (8) and (9) into Eq. (7) results in a quadratic equation as

$S_{1} z_{\mathrm{C}}^{2}+S_{2} z_{\mathrm{C}}+S_{3}=0$,

where $S_{1}=Q^{2}+N^{2}+1, S_{2}=2(P Q+M N)$ and $S_{3}=P^{2}+M^{2}-1$.

Solving Eq. (10), the coordinate of $z_{C}$ can be obtained as

$z_{\mathrm{C}}=\frac{-S_{2} \pm \sqrt{S_{2}^{2}-4 S_{1} S_{3}}}{2 S_{1}}$.

Thus, the value of joint angle $\theta_{4}$ is obtained by substituting Eq. (11) into $z_{\mathrm{C}}$ of Eq. (3) as

$\theta_{4}=-\operatorname{arcc}\left(\cot \varphi_{3} \cot \varphi_{4}-z_{\mathrm{C}} / s \varphi_{3} s \varphi_{4}\right)$.

The two possible values of $z_{\mathrm{C}}$ result in two joint angle $\theta_{4}$, leading to two configurations of $\triangle \mathrm{BCD}$, one of which represents the case that the triangle vertex $\mathrm{C}$ appears above $\mathrm{BD}$ and the other indicates the case when vertex $C$ is below $B D$.

Apart from Eq. (3), the position vector of point $\mathrm{C}$ can be expressed in another form as 


$$
\boldsymbol{P}_{\mathrm{C}}=\mathbf{R}\left(y_{5}, \varphi_{5}\right) \mathbf{R}\left(z_{1}, \theta_{1}\right) \mathbf{R}\left(y_{1}, \varphi_{1}\right) \mathbf{R}\left(z_{2}, \theta_{2}\right) \mathbf{R}\left(y_{2}, \varphi_{2}\right) \boldsymbol{P}_{\mathrm{E}}
$$

which leads to another expression of $z_{\mathrm{C}}$ as

$$
\begin{aligned}
z_{\mathrm{C}}= & c \varphi_{2}\left(c \varphi_{1} c \varphi_{5}-s \varphi_{1} s \varphi_{5} c \theta_{1}\right) \\
& -s \varphi_{2}\left(\left(s \varphi_{1} c \varphi_{5}+c \varphi_{1} s \varphi_{5} c \theta_{1}\right) c \theta_{2}-s \varphi_{5} s \theta_{1} s \theta_{2}\right),
\end{aligned}
$$

Substituting Eq. (14) into Eq. (11) and rearranging the equation yields

$T_{1} c \theta_{2}+T_{2} s \theta_{2}-T_{3}=0$,

where,

$$
\begin{aligned}
T_{1}= & s \varphi_{2}\left(s \varphi_{1} c \varphi_{5}-c \varphi_{1} s \varphi_{5} c \theta_{1}\right), \\
T_{2}= & s \varphi_{2} s \varphi_{5} s \theta_{1} \text { and } T_{3}=c \varphi_{5}\left(c \varphi_{1} c \varphi_{5}+s \varphi_{1} s \varphi_{5} c \theta_{1}\right) \\
& +\left(S_{2} \mp \sqrt{S_{2}^{2}-4 S_{1} S_{3}}\right) / 2 S_{1} .
\end{aligned}
$$

Solving Eq. (15) gives the joint angle $\theta_{2}$ as

$\theta_{2}=\arctan \frac{T_{2}}{T_{1}} \pm \operatorname{arccot}\left(\frac{T_{3}}{\sqrt{T_{1}^{2}+T_{2}^{2}}}\right)$.

The above equation indicates two solutions for $\theta_{2}$, one of which implies the triangle vertex B locates below $\mathrm{AC}$, and the other represents vertex $\mathrm{B}$ above AC. Because the reconfigurable base is a closed chain, the joint value $\theta_{1}$ and $\theta_{5}$ are not totally independent. When assigning the value of angle $\theta_{5}$, the spherical five-bar linkage mechanism degenerates to a spherical four-bar linkage mechanism. At that time, rotating joint A will make the spherical four-bar linkage mechanism reach its limited positions when point $\mathrm{B}, \mathrm{C}$ and $\mathrm{D}$ lie in the same plane, thus it has,

$\boldsymbol{P}_{\mathrm{D}} \cdot\left(\boldsymbol{P}_{\mathrm{B}} \times \boldsymbol{P}_{\mathrm{C}}\right)=0$,

$\boldsymbol{P}_{\mathrm{B}} \cdot\left(\boldsymbol{P}_{\mathrm{D}} \times \boldsymbol{P}_{\mathrm{C}}\right)=0$.

The mechanism has two limited position as the link $\mathrm{AD}$ can rotate on both side with respect to link DC. Thus, the value $\theta_{5}$ decides the range of that of $\theta_{1}$, the relation between the two angles can be obtained as

$\boldsymbol{P}_{\mathrm{B}}^{\mathrm{T}} \cdot \boldsymbol{P}_{\mathrm{A}}=c \varphi_{1}$,

$\boldsymbol{P}_{\mathrm{B}}^{\mathrm{T}} \cdot \boldsymbol{P}_{\mathrm{D}}=c\left(\varphi_{2}+\varphi_{3}\right)$,

$\boldsymbol{P}_{\mathrm{B}}^{\mathrm{T}} \cdot \boldsymbol{P}_{\mathrm{B}}=1$.
Substituting Eqs. (1), (2) and (3) into Eqs. (19) throughout (21) and solving the latter gives the two limited values of angle $\theta_{1}$ as

$\theta_{l \lim }=\operatorname{arct}\left(\frac{U_{2}}{U_{1}}\right) \pm \operatorname{arcc}\left(\frac{-U_{3}}{\sqrt{U_{1}^{2}+U_{2}^{2}}}\right)$,

where $\quad U_{1}=-s \varphi_{4} c \theta_{5} c \varphi_{5} \mathrm{~s} \varphi_{1}-s \varphi_{1} c \varphi_{4} s \varphi_{5}, U_{2}=$ $s \varphi_{1} s \varphi_{4} s \theta_{5}$ and $U_{3}=c\left(\varphi_{2}+\varphi_{3}\right)-c \varphi_{1} c \varphi_{4} c \theta_{5}+s \varphi_{4}$ $c \varphi_{1} c \theta_{5} s \varphi_{5}$.

Hence the range of $\theta_{1}$ with respect to $\theta_{5}$ is

$V_{1} \leq \theta_{l} \leq V_{2}$,

where

$$
\begin{aligned}
& V_{1}=\operatorname{arct}\left(\frac{U_{2}}{U_{1}}\right)-\operatorname{arcs}\left(\frac{-U_{2}}{\sqrt{U_{1}^{2}+U_{2}^{2}}}\right) \text { and } \\
& V_{2}=\operatorname{arct}\left(\frac{U_{2}}{U_{1}}\right)+\operatorname{arcc}\left(\frac{-U_{3}}{\sqrt{U_{1}^{2}+U_{2}^{2}}}\right)
\end{aligned}
$$

Based on the above analysis, it can be found that given a pair of $\theta_{1}$ and $\theta_{5}$, there are four groups of solution for $\theta_{2}, \theta_{3}$ and $\theta_{4}$ resulting in four different configurations of the base. Motion planning is needed when controlling this mechanism because the configuration of spherical base is considered by the order of its inputs.

\subsection{Position of the 3-RRS parallel mechanism in a particular configuration of the reconfigurable base}

A local coordinate system $\mathrm{M}^{\prime}\left\{\mathrm{O}^{\prime}-x^{\prime} y^{\prime} z^{\prime}\right\}$ is attached to the upper moving platform with the origin $\mathrm{O}^{\prime}$ coincided with the centroid of the equilateral triangle $\Delta \mathrm{C}_{1} \mathrm{C}_{2} \mathrm{C}_{3}$ and the $z^{\prime}$-axis directed to point $\mathrm{C}_{1}$. The coordinates of $\mathrm{A}_{i}(i=1,2$ and 3$)$ in the global coordinate system are given by,

$$
\begin{aligned}
{ }^{\mathrm{F}} \boldsymbol{P}_{\mathrm{A}_{1}}= & {\left[\begin{array}{lll}
0 & 0 & 1
\end{array}\right]^{\mathrm{T}}, } \\
{ }^{\mathrm{F}} \boldsymbol{P}_{\mathrm{A}_{2}}= & \mathbf{R}\left(y, \delta_{1}\right) \mathbf{R}\left(z_{1}, \theta_{1}\right) \mathbf{R}\left(y_{1}, \varphi_{1}\right) \mathbf{R}\left(z_{2}, \theta_{2}\right) \mathbf{R}\left({ }_{2}\right) \\
{ }^{\mathrm{F}} \boldsymbol{P}_{\mathrm{A}_{3}}= & \mathbf{R}\left(y,-\delta_{1}\right) \mathbf{R}\left(z_{5},-\theta_{5}\right) \mathbf{R}\left(y_{4},-\varphi_{4}\right) \\
& \mathbf{R}\left(z_{4},-\theta_{4}\right) \mathbf{R}\left(y_{3},-\delta_{3}\right){ }^{\mathrm{F}} \boldsymbol{P}_{\mathrm{A}_{1}},
\end{aligned}
$$$$
{ }^{\mathrm{F}} \boldsymbol{P}_{\mathrm{A}_{2}}=\mathbf{R}\left(y, \delta_{1}\right) \mathbf{R}\left(z_{1}, \theta_{1}\right) \mathbf{R}\left(y_{1}, \varphi_{1}\right) \mathbf{R}\left(z_{2}, \theta_{2}\right) \mathbf{R}\left(y_{2}, \delta_{2}\right){ }^{\mathrm{F}} \boldsymbol{P}_{\mathrm{A}_{1}},
$$

The coordinates of $\mathrm{C}_{i}$ in the coordinate system $\mathrm{M}^{\prime}$ can be obtained as 
${ }^{\mathrm{M}} \boldsymbol{P}_{\mathrm{C}_{1}}=\left[\begin{array}{lll}0 & 0 & r\end{array}\right]^{\mathrm{T}}$,

${ }^{\mathrm{M}^{\prime}} \boldsymbol{P}_{\mathrm{C}_{2}}=\left[\begin{array}{lll}\frac{\sqrt{3}}{2} r & 0 & -\frac{r}{2}\end{array}\right]^{\mathrm{T}}$,

${ }^{\mathrm{M}^{\prime}} \boldsymbol{P}_{\mathrm{C}_{3}}=\left[\begin{array}{lll}-\frac{\sqrt{3}}{2} r & 0 & -\frac{r}{2}\end{array}\right]^{\mathrm{T}}$,

The upper moving platform $\mathrm{C}_{1} \mathrm{C}_{2} \mathrm{C}_{3}$ is an equilateral triangle as $\left|O^{\prime} C_{1}\right|=\left|O^{\prime} C_{2}\right|=\left|O^{\prime} C_{3}\right|=3 r^{2}$. The position vector ${ }^{\mathrm{F}} \boldsymbol{P}_{\mathrm{C} i}$ of $\mathrm{C}_{i}(i=1,2$ and 3$)$ with respect to global coordinate frame $\mathrm{F}$ is given by the transformation as follows,

${ }^{\mathrm{F}} \boldsymbol{P}_{\mathrm{C} i}={ }^{\mathrm{F}} \boldsymbol{P}_{\mathrm{OO}^{\prime}}+{ }^{\mathrm{F}} \mathbf{R}_{\mathrm{M}^{\prime}}{ }^{\mathrm{M}^{\prime}} \boldsymbol{P}_{\mathrm{C} i},(i=1,2$ and 3$)$,

where ${ }^{\mathrm{F}} \boldsymbol{P}_{\mathrm{OO}^{\prime}}$ is the position vector of $\mathrm{O}^{\prime}$ expressed in the global coordinate frame $\mathrm{F}$ and ${ }^{\mathrm{F}} \mathbf{R}_{\mathrm{M}^{\prime}}$ is the rotation matrix indicating the rotation of coordinates from coordinate frame $\mathbf{M}^{\prime}$ to the global coordinate frame F.

\subsection{Forward kinematics of the spherical-base integrated parallel mechanism}

The sequence of calculating the forward kinematics of the spherical-base integrated mechanism is to take the configuration of the base into consideration primarily as a way to degenerate the whole mechanism into a 3-RRS mechanism with a confirmed base configuration, then apply the way to formulating forward kinematics of a parallel mechanism to this simplified parallel mechanism, which is well presented in the Ref. [35-37]. For each limb in this proposed mechanism, the local limb coordinate system $\mathrm{K}_{\mathrm{i}}\left\{\mathrm{O}-x_{K i} y_{K i} z_{K i}\right\}$ ( $i=1,2$ and 3 ) is established with the origin $\mathrm{O}, z_{K i}$-axis directed to point $\mathrm{A}_{i}$ and $y_{K i}$-axis perpendicular to the plane formed by the linkage of the reconfigurable base. In terms of Fig. 4 , the $y_{K 2}$-axis is perpendicular to the plane constructed by $\triangle \mathrm{COD}$.

The position vector of point $C_{i}$ in the global coordinate frame can be described as

$\boldsymbol{k}_{\boldsymbol{i}}=\mathbf{R}_{\mathbf{K} i}\left[\begin{array}{c}0 \\ l_{i} s \alpha_{i} \\ 1-l_{i} c \alpha_{i}\end{array}\right], \quad i=1,2,3$,

where $\boldsymbol{k}_{i}$ is the position vector of point $\mathrm{C}_{i}$ expressed in the local coordinate system $\mathrm{M}_{5}\left\{\mathrm{O}-x_{5} y_{5} z_{5}\right\}, \mathbf{R}_{\mathbf{K} i}$

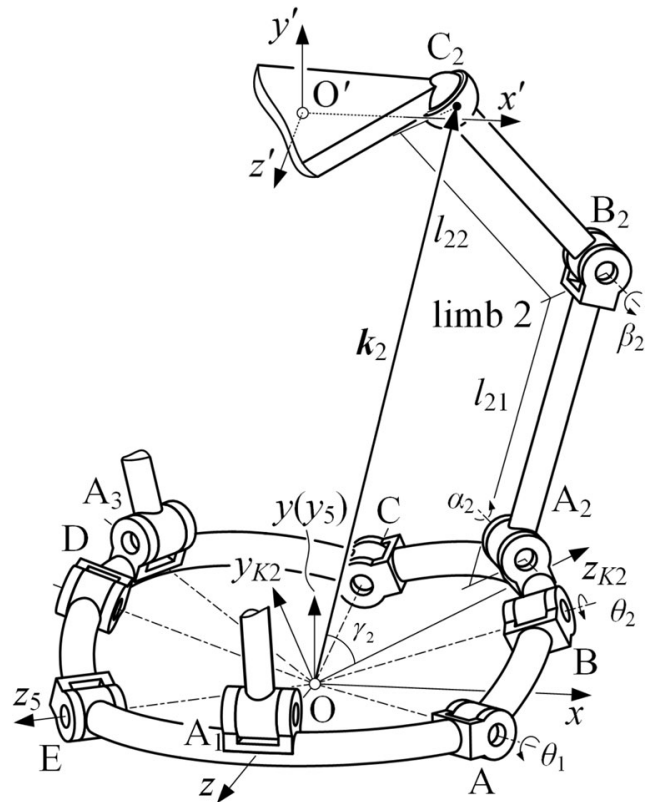

Fig. 4 Kinematic analysis for limb 2 of the spherical-base integrated parallel mechanism

describes the transformation from the local limb coordinate system to the coordinate system $\mathrm{M}_{5}\left\{\mathrm{O}-x_{5} y_{5} z_{5}\right\}$ as

$$
\begin{aligned}
\mathbf{R}_{\mathbf{K} i}= & {\left[\begin{array}{lll}
u_{x i} & v_{x i} & w_{x i} \\
u_{y i} & v_{y i} & w_{y i} \\
u_{z i} & v_{z i} & w_{z i}
\end{array}\right] } \\
= & \begin{cases}\mathbf{R}\left(y, \delta_{1}\right), & i=1 \\
\mathbf{R}\left(y, \varphi_{5}\right) \mathbf{R}\left(z, \theta_{1}\right) \mathbf{R}\left(y, \varphi_{1}\right) \mathbf{R}\left(z, \theta_{2}\right) \mathbf{R}\left(y, \delta_{2}\right), & i=2, \\
\mathbf{R}\left(z,-\theta_{5}\right) \mathbf{R}\left(y,-\varphi_{4}\right) \mathbf{R}\left(z,-\theta_{4}\right) \mathbf{R}\left(y,-\delta_{3}\right), & i=3\end{cases} \\
& i=1,2 \text { and } 3
\end{aligned}
$$

The values of $\theta_{2}$ and $\theta_{4}$ can be obtained through Eqs. (16) and (12) according to the geometry constraints of the reconfigurable base. So $\boldsymbol{k}_{i}$ is computed by substituting $\theta_{2}$ and $\theta_{4}$ together with Eq. (32) into Eq. (31) as

$$
\begin{aligned}
\boldsymbol{k}_{1} & =\left[\begin{array}{c}
\left(1-l_{1} c \alpha_{1}\right) s \delta_{1} \\
l_{1} s \alpha_{1} \\
\left(1-l_{1} c \alpha_{1}\right) c \delta_{1}
\end{array}\right], \boldsymbol{k}_{i} \\
& =\left[\begin{array}{l}
w_{x i}\left(1-l_{i} c \alpha_{i}\right)+l_{i} v_{x i} \mathrm{~s} \alpha_{i} \\
w_{y i}\left(1-l_{i} c \alpha_{i}\right)+l_{i} v_{y i} \mathrm{~s} \alpha_{i} \\
w_{z i}\left(1-l_{i} c \alpha_{i}\right)+l_{i} v_{z i} \mathrm{~s} \alpha_{i}
\end{array}\right], \\
i & =2 \text { and } 3 .
\end{aligned}
$$


For calculating the forward kinematics, the angle value of joint $\mathrm{B}_{i}$ is given, hence the length between point $\mathrm{A}_{i}$ and $\mathrm{C}_{i}$ is introduced by the following equation,

$l_{i}=\sqrt{l_{i 1}^{2}+l_{i 2}^{2}-2 l_{i 1} l_{i 2} c \beta_{i}}, \quad i=1,2$ and 3.

Thus, the upper moving platform, which is connected to the limbs by spatial joints, restricts the position of point $C_{i}$; that is

$$
\left(\boldsymbol{k}_{i}-\boldsymbol{k}_{i+1}\right)^{\mathrm{T}}\left(\boldsymbol{k}_{i}-\boldsymbol{k}_{i+1}\right)=3 r^{2}, \quad i=1,2 \text { and } 3 .
$$

Equation (35) describes the geometrical relation between the endpoints of any two limbs. So if $i=3$, then $i+1$ is equal to 1 . By utilizing the way to calculating forward kinematics of the parallel mechanism introduced by Merlet [36], we can see that there are up to 16 solutions for the fixed-base parallel mechanism. However, in this spherical-base integrated mechanism, the number doubles as the reconfigurable base provides two configurations for given inputs investigated in Sect. 3.1. Path planning is necessary to get a desired configuration of the base and the moving platform.

The workspace of the spherical-base integrated parallel mechanism proposed in this paper is larger than that of the 3-RRS parallel mechanism with same limb and platform constructions and parameters. To make the comparison fairly, the base of the 3-RRS parallel mechanism is designed as the same of the initial configuration of the proposed mechanism (stated in Sect. 2.2) Under this definition, the workspace of this proposed mechanism is enlarged compared with the 3-RRS fixed-base parallel mechanism. By locking the base of this proposed mechanism in its initial state, it shares the same workspace of the 3-RRS parallel mechanism. When the joints of the base are released, it will contribute to a larger workspace as you can always lock the base during its motion in where the mechanism degenerates to a 3-RRS mechanism as a consequence. In other words, the workspace of the spherical-base integrated parallel mechanism is the sum of the workspaces of its degenerated 3-RRS parallel mechanisms with all possible base configurations.

\section{Inverse kinematics of the spherical-base integrated parallel mechanism}

The inverse kinematic problem can be described as giving the position and orientation of the upper moving platform to acquire the rotation angle of each active joint. For simplifying the model, we assume that point $\mathrm{A}_{i}(i=1,2$ and 3$)$ is on the links of the reconfigurable base. By decomposing the hybrid mechanism into a spherical five-bar linkage and a 3-RRS parallel mechanism where its three limbs are mounted on the former, the inverse kinematics of each linkage is investigated and then integrated through the instinct geometry when connecting them. As the configurations of the reconfigurable five-bar base plays a vital role in deciding the position and orientation of the platform, it has to be primarily considered. In this case, the procedures of obtaining the inverse kinematics of this mechanism are divided into the following two steps:

1. Find all possible configurations of the base relying on its geometric constraints with the platform when the position and orientation of the latter is given.

2. For considering the mechanism as a whole after decouple, the length of each limb has to be achievable which it lies in the certain boundary.

For every possible configuration of the spherical base, it has to satisfy the geometric condition that the plane constructed by $\Delta \mathrm{OA}_{i} \mathrm{~B}_{i}(i=1,2$ or 3$)$ is perpendicular to its corresponding plane constructed by the linkage of the reconfigurable base. As shown in Fig. $4, \Delta \mathrm{OA}_{2} \mathrm{~B}_{2}$ is orthogonal to $\triangle \mathrm{OBC}$. Denote $C_{i}^{\prime}$ the intersection between $\mathrm{OC}_{i}$ and the surface of the sphere constructed by the spherical mechanism. In this way, the coordinates of $C_{3}^{\prime}$ can be written as,

$$
\begin{aligned}
\boldsymbol{P}_{C_{3}^{\prime}} & =\frac{1}{\sqrt{x_{C_{3}}^{2}+y_{C_{3}}^{2}+z_{C_{3}}^{2}}}\left[\begin{array}{lll}
x_{C_{3}} & y_{C_{3}} & z_{C_{3}}
\end{array}\right]^{\mathrm{T}} \\
& =\left[\begin{array}{lll}
x_{C_{3}^{\prime}} & y_{C_{3}^{\prime}} & z_{C_{3}^{\prime}}^{\prime}
\end{array}\right]^{\mathrm{T}}
\end{aligned}
$$

The coordinates of $C_{3}^{\prime}$ can be acquired in another way by multiplying the rotation matrices from the point $\mathrm{E}$ as, 


$$
\begin{aligned}
& \boldsymbol{P}_{\mathrm{C}}=\mathbf{R}\left(z_{5},-\theta_{5}\right) \mathbf{R}\left(y_{4},-\varphi_{4}\right) \mathbf{R}\left(z_{4},-\theta_{4}\right) \mathbf{R}\left(y_{3},-\delta_{3}\right) \mathbf{R}\left(x_{3},-\gamma_{3}\right) \boldsymbol{P}_{\mathrm{E}} \\
& =\left[\begin{array}{c}
c \gamma_{3}\left(s \delta_{3}\left(s \theta_{4} s \theta_{5}-c \varphi_{4} c \theta_{4} c \theta_{5}\right)-c \delta_{3} c \theta_{5} s \varphi_{4}\right)+s \gamma_{3}\left(c \theta_{4} s \theta_{5}+c \varphi_{4} c \theta_{5} s \theta_{4}\right) \\
c \gamma_{3}\left(s \delta_{3}\left(s \theta_{4} c \theta_{5}+c \varphi_{4} c \theta_{4} s \theta_{5}\right)+c \delta_{3} s \theta_{5} s \varphi_{4}\right)+s \gamma_{3}\left(c \theta_{4} c \theta_{5}-c \varphi_{4} s \theta_{4} s \theta_{5}\right) \\
c \gamma_{3}\left(c \delta_{3} c \varphi_{4}-s \delta_{3} c \theta_{4} s \varphi_{4}\right)+s \gamma_{3} s \varphi_{4} s \theta_{4}
\end{array}\right]=\left[\begin{array}{l}
x_{\mathrm{C}_{3}^{\prime}} \\
y_{\mathrm{C}_{3}^{\prime}} \\
z_{\mathrm{C}_{3}^{\prime}}
\end{array}\right]
\end{aligned}
$$

As there are only three unknowns $\theta_{4}, \theta_{5}$ and $r_{3}$ in Eq. (36), the standard trigonometric substitutions are adopted to estimate these unknown variables,

$$
\left\{\begin{array}{l}
s \gamma_{3}=\frac{2 u_{1}}{1+u_{1}^{2}}, c \gamma_{r}=\frac{1-u_{1}^{2}}{1+u_{1}^{2}} \\
s \theta_{4}=\frac{2 u_{2}}{1+u_{2}^{2}}, c \theta_{4}=\frac{1-u_{1}^{2}}{1+u_{1}^{2}} \\
s \theta_{5}=\frac{2 u_{3}}{1+u_{3}^{2}}, c \theta_{5}=\frac{1-u_{3}^{2}}{1+u_{3}^{2}}
\end{array}\right.
$$

In order to estimate $u_{2}$, substituting Eq. (38) into the equations in Eq. (37) results in three quadratic equations in $u_{2}$

$E_{1} u_{2}^{2}+E_{2} u_{2}+E_{3}=0$

$F_{1} u_{2}^{2}+F_{2} u_{2}+F_{3}=0$,

$G_{1} u_{2}^{2}+G_{2} u_{2}+G_{3}=0$,

where $E_{i}$ and $F_{i}$ are second-degree polynomials in $u_{3}$ and $u_{1}$ and $G_{i}(i=1,2,3)$ is the second-degree polynomials in $u_{1}$, shown in Appendix 1. Sylvester Dialytic Elimination Method [38] can be utilized here to estimate $u_{2}$ from these equations. Taking Eq. (38) $\times G_{1}-$ Eq. (40) $\times E_{1}$ and Eq. (38) $\times$ $G_{3}-$ Eq. (40) $\times E_{3}$ respectively, the two linear equation in $u_{3}$ are obtained as

$$
\begin{aligned}
& \left(E_{1} G_{2}-E_{2} G_{1}\right) u_{2}+\left(E_{1} G_{3}-E_{3} G_{1}\right)=0 \\
& \left(E_{3} G_{1}-E_{1} G_{3}\right) u_{2}+\left(E_{3} G_{2}-E_{2} G_{3}\right)=0
\end{aligned}
$$

Combining Eqs. (41) and (42) to estimate $u_{2}$, we obtain,

$\left(E_{1} G_{3}-E_{3} G_{1}\right)^{2}+\left(E_{1} G_{2}-E_{2} G_{1}\right)\left(E_{3} G_{2}-E_{2} G_{3}\right)=0$
A fourth-degree polynomial in $u_{3}$ can be derived from substituting $E_{i}$ and $G_{i}(i=1,2,3)$ into Eq. (43); that is,

$J_{1} u_{3}^{4}+J_{2} u_{3}^{3}+J_{3} u_{3}^{2}+J_{4} u_{3}+J_{5}=0$

where $J_{i}(i=1$ to 5$)$ is eighth-degree polynomials in $u_{1}$. Taking the same method for Eqs. (39) and (40), and writing it in the similar form with Eq. (44) as,

$H_{1} u_{3}^{4}+H_{2} u_{3}^{3}+H_{3} u_{3}^{2}+H_{4} u_{3}+H_{5}=0$

where $H_{i}(i=1$ to 3$)$ is eighth-degree polynomials in $u_{1}$. For estimating the term $u_{3}^{4}$, we multiply Eq. (44) by $H_{1}$ and Eq. (45) by $J_{1}$, and then take the subtraction of the two to obtain the a third-degree polynomial in $u_{3}$, as follows,

$$
\begin{aligned}
& \left(H_{2} J_{1}-H_{1} J_{2}\right) u_{3}^{3}+\left(H_{3} J_{1}-H_{1} J_{3}\right) u_{3}^{2} \\
& \quad+\left(H_{4} J_{1}-H_{1} J_{4}\right) u_{3}+\left(H_{5} J_{1}-H_{1} J_{5}\right)=0
\end{aligned}
$$

Another equation of $u_{3}$ can be derived from multiplying Eq. (44) by $\mathrm{H}_{2}$ and Eq. (45) by $J_{2}$, and subtracting, which yields,

$$
\begin{aligned}
& \left(H_{2} J_{1}-H_{1} J_{2}\right) u_{3}^{4}+\left(H_{2} J_{3}-H_{3} J_{2}\right) u_{3}^{2} \\
& +\left(H_{2} J_{4}-H_{4} J_{2}\right) u_{3}+\left(H_{2} J_{5}-H_{5} J_{2}\right)=0
\end{aligned}
$$

Multiplying Eq. (45) by $u_{3}$ leads to

$$
\begin{aligned}
& \left(H_{2} J_{1}-H_{1} J_{2}\right) u_{3}^{4}+\left(H_{3} J_{1}-H_{1} J_{3}\right) u_{3}^{3} \\
& +\left(H_{4} J_{1}-H_{1} J_{4}\right) u_{3}^{2}+\left(H_{5} J_{1}-H_{1} J_{5}\right) u_{3}=0
\end{aligned}
$$

We write Eqs. (44) throughout (48) in matrix form as

$\mathbf{M} \cdot\left[u_{3}^{4}, u_{3}^{3}, u_{3}^{2}, u_{3}, 1\right]^{T}=\mathbf{0}$

where 
$\mathbf{M}=\left[\begin{array}{ccccc}H_{1} & H_{2} & H_{3} & H_{4} & H_{5} \\ J_{1} & J_{2} & J_{3} & J_{4} & J_{5} \\ H_{2} J_{1}-H_{1} J_{2} & H_{3} J_{1}-H_{1} J_{3} & H_{4} J_{1}-H_{1} J_{4} & H_{5} J_{1}-H_{1} J_{5} & 0 \\ H_{2} J_{1}-H_{1} J_{2} & 0 & H_{2} J_{3}-H_{3} J_{2} & H_{2} J_{4}-H_{4} J_{2} & H_{2} J_{5}-H_{5} J_{2} \\ 0 & H_{2} J_{1}-H_{1} J_{2} & H_{3} J_{1}-H_{1} J_{3} & H_{4} J_{1}-H_{1} J_{4} & H_{5} J_{1}-H_{1} J_{5}\end{array}\right]$

Equation (49) is valid if and only if the determinant of $\mathbf{M}$ is equal to zero, which results in a thirty-seconddegree polynomial in the square of $u_{1}$, as follow,

$\sum_{i=0}^{64} N_{i} u_{1}^{i}=0$

In Eq. (50), $N_{i}$ is only decided by the structure of the hybrid mechanism. Solving Eq. (50) will obtain at most 32 pairs of solutions for $u_{1}$, with each pair containing one positive and one negative solution. The other two parameters $u_{2}$ and $u_{3}$ can be calculated by substituting $u_{1}$ into Eqs. (45) and (40) separately, which overall results in 64 groups of solution for $u_{2}$ and $u_{3}$. Thus, $\theta_{4}, \theta_{5}$ and $r_{3}$ are solved in the Eq. (38) by substituting the above parameter into these trigonometric functions. Once $\theta_{4}$ and $\theta_{5}$ are obtained, the configuration of the spherical base is confirmed. The next step is to solve the configuration for the limbs based on the known base configuration and moving platform.

With explicit expressions of $\boldsymbol{P}_{\mathrm{Ai}}$ and $\boldsymbol{k}_{\mathrm{i}}(i=1,2$ or 3 ), we can move forward to obtain the length of each limb so as to get the value of $\beta_{i}(i=1,2$ or 3$)$ in the $\Delta \mathrm{A}_{i} \mathrm{~B}_{i} \mathrm{C}_{i}$ and complete to solve the inverse kinematics, by the following equation,

$c \beta_{i}=\left(l_{i 1}^{2}+l_{i 2}^{2}-l_{i}^{2}\right) / 2 l_{i 1} l_{i 2}, \quad i=1,2$ and 3.

Two solutions of $\beta_{i}(i=1,2$ or 3 ) exist for nonsingular configurations of each limb that contribute to 8 possible states for a specific base configuration, which leads to at most 512 different configurations for the whole mechanism theoretically. The amount of possible configurations is much smaller that the theoretical number because the solutions of the spherical base only consider the geometrical constraint of limb 3, and these solutions need to be verified to satisfy the corresponding geometrical constraint of limb 2, which will significantly decrease the number of solutions.

\section{Screw theory based Jacobian analysis}

As aforementioned, since the parallel mechanism consists of three limbs mounted on a reconfigurable base, structure decomposition of the mechanism can be applied to analysing the Jacobian matrix of the whole mechanism, the Jacobian analysis of the reconfigurable base can be completed first followed by that of the parallel mechanism.

\subsection{Jacobian analysis for the reconfigurable base}

The velocity of point $\mathrm{C}$ can be expressed as a linear combination of angular velocity of axis $\mathrm{OA}$ and $\mathrm{OB}$ or the other linear combination of angular velocity of axis $\mathrm{OE}$ and OD,

$$
\begin{aligned}
& \boldsymbol{v}_{\mathrm{C}}=\dot{\theta}_{1}\left(\boldsymbol{P}_{\mathrm{A}} \times \boldsymbol{P}_{\mathrm{C}}\right)+\dot{\theta}_{2}\left(\boldsymbol{P}_{\mathrm{B}} \times \boldsymbol{P}_{\mathrm{C}}\right), \\
& \boldsymbol{v}_{\mathrm{C}}=\dot{\theta}_{5}\left(\boldsymbol{P}_{\mathrm{E}} \times \boldsymbol{P}_{\mathrm{C}}\right)+\dot{\theta}_{4}\left(\boldsymbol{P}_{\mathrm{D}} \times \boldsymbol{P}_{\mathrm{C}}\right) .
\end{aligned}
$$

Since $v_{C}$ is a passive variable, it should be estimated from Eqs. (52) and (53). Thus we take right inner product on both side of Eqs. (52) and (53) with $\boldsymbol{P}_{\mathrm{D}}$, it has

$$
\begin{aligned}
& \boldsymbol{P}_{\mathrm{D}} \cdot \boldsymbol{v}_{\mathrm{C}}=\dot{\theta}_{1} \boldsymbol{P}_{\mathrm{D}} \cdot\left(\boldsymbol{P}_{\mathrm{A}} \times \boldsymbol{P}_{\mathrm{C}}\right)+\dot{\theta}_{2} \boldsymbol{P}_{\mathrm{D}} \cdot\left(\boldsymbol{P}_{\mathrm{B}} \times \boldsymbol{P}_{\mathrm{C}}\right), \\
& \boldsymbol{P}_{\mathrm{D}} \cdot \boldsymbol{v}_{\mathrm{C}}=\dot{\theta}_{5} \boldsymbol{P}_{\mathrm{D}} \cdot\left(\boldsymbol{P}_{\mathrm{E}} \times \boldsymbol{P}_{\mathrm{C}}\right) .
\end{aligned}
$$

Substituting Eq. (55) into Eq. (54) yields, $\dot{\theta}_{2}=-\frac{\boldsymbol{P}_{\mathrm{D}} \cdot\left(\boldsymbol{P}_{\mathrm{A}} \times \boldsymbol{P}_{\mathrm{C}}\right)}{\boldsymbol{P}_{\mathrm{D}} \cdot\left(\boldsymbol{P}_{\mathrm{B}} \times \boldsymbol{P}_{\mathrm{C}}\right)} \dot{\theta}_{1}+\frac{\boldsymbol{P}_{\mathrm{D}} \cdot\left(\boldsymbol{P}_{\mathrm{E}} \times \boldsymbol{P}_{\mathrm{C}}\right)}{\boldsymbol{P}_{\mathrm{D}} \cdot\left(\boldsymbol{P}_{\mathrm{B}} \times \boldsymbol{P}_{\mathrm{C}}\right)} \dot{\theta}_{5}$.

Similarly, the angular velocity $\dot{\theta}_{4}$ can be obtained and expressed as, 
$\dot{\theta}_{4}=\frac{\boldsymbol{P}_{\mathrm{B}} \cdot\left(\boldsymbol{P}_{\mathrm{A}} \times \boldsymbol{P}_{\mathrm{C}}\right)}{\boldsymbol{P}_{\mathrm{B}} \cdot\left(\boldsymbol{P}_{\mathrm{D}} \times \boldsymbol{P}_{\mathrm{C}}\right)} \dot{\theta}_{1}-\frac{\boldsymbol{P}_{\mathrm{B}} \cdot\left(\boldsymbol{P}_{\mathrm{E}} \times \boldsymbol{P}_{\mathrm{C}}\right)}{\boldsymbol{P}_{\mathrm{B}} \cdot\left(\boldsymbol{P}_{\mathrm{D}} \times \boldsymbol{P}_{\mathrm{C}}\right)} \dot{\theta}_{5}$.

The Eqs. (56) and (57) can be expressed in matrix form as,

$$
\begin{aligned}
{\left[\begin{array}{c}
\dot{\theta}_{2} \\
\dot{\theta}_{4}
\end{array}\right]=} & \boldsymbol{J}_{\varphi}\left[\begin{array}{c}
\dot{\theta}_{1} \\
\dot{\theta}_{5}
\end{array}\right] \\
= & {\left[\begin{array}{cc}
-\frac{\boldsymbol{P}_{\mathrm{D}} \cdot\left(\boldsymbol{P}_{\mathrm{A}} \times \boldsymbol{P}_{\mathrm{C}}\right)}{\boldsymbol{P}_{\mathrm{D}} \cdot\left(\boldsymbol{P}_{\mathrm{B}} \times \boldsymbol{P}_{\mathrm{C}}\right)} & \frac{\boldsymbol{P}_{\mathrm{D}} \cdot\left(\boldsymbol{P}_{\mathrm{E}} \times \boldsymbol{P}_{\mathrm{C}}\right)}{\boldsymbol{P}_{\mathrm{D}} \cdot\left(\boldsymbol{P}_{\mathrm{B}} \times \boldsymbol{P}_{\mathrm{C}}\right)} \\
\frac{\boldsymbol{P}_{\mathrm{B}} \cdot\left(\boldsymbol{P}_{\mathrm{A}} \times \boldsymbol{P}_{\mathrm{C}}\right)}{\boldsymbol{P}_{\mathrm{B}} \cdot\left(\boldsymbol{P}_{\mathrm{D}} \times \boldsymbol{P}_{\mathrm{C}}\right)} & -\frac{\boldsymbol{P}_{\mathrm{B}} \cdot\left(\boldsymbol{P}_{\mathrm{E}} \times \boldsymbol{P}_{\mathrm{C}}\right)}{\boldsymbol{P}_{\mathrm{B}} \cdot\left(\boldsymbol{P}_{\mathrm{D}} \times \boldsymbol{P}_{\mathrm{C}}\right)}
\end{array}\right]\left[\begin{array}{c}
\dot{\theta}_{1} \\
\dot{\theta}_{5}
\end{array}\right] . }
\end{aligned}
$$

Thus the angular velocity of passive joints $\mathrm{B}$ and $\mathrm{D}$ is calculated through that of active joints $\mathrm{A}$ and $\mathrm{E}$ based on the geometric constraints of the spherical mechanism.

\subsection{Jacobian analysis for the spherical-base integrated parallel mechanism}

The screw algebra is introduced in this section for analysing the velocity of the spherical-base integrated parallel mechanism. A screw $\boldsymbol{S}$ is a six-dimensional vector representing instantaneous kinematic properties of a rigid body, commonly expressed as,

$\boldsymbol{S}=\left[\begin{array}{c}\boldsymbol{s} \\ \boldsymbol{s}_{0}\end{array}\right]=\left[\begin{array}{c}\boldsymbol{s} \\ \boldsymbol{r} \times \boldsymbol{s}+h \boldsymbol{s}\end{array}\right]=\left[s_{x}, s_{y}, s_{z}, s_{x 0}, s_{y 0}, s_{z 0}\right]^{\mathrm{T}}$.

The first three components consist of a unit vector $\boldsymbol{s}$ directing along the screw axis, as well as the joint axis when describing a rotation. The last elements constitute $s_{0}$ representing the moment of the vector $s$ about the origin of the reference frame, and $h$ expresses the screw pitch, which is equal to 0 for revolute joints and $\infty$ for prismatic joints, $\boldsymbol{r}$ is the position vector from the origin of the reference coordinate system to an arbitrary point on the screw axis $\boldsymbol{s}$.

The whole mechanism Jacobian can be derived from the twist of the mechanism based on screw system notation. Figure 5a illustrates motion screws of the spherical-base integrated parallel mechanism. We can assume each limb as an open-loop chain connecting the end-effector to the base, as shown in Fig. 5b. Defining $S_{p}$ as the instantaneous motion of the moving platform, the twist $S_{p}$ can be derived from the linear combination of each joint's twist within the loop. Referring to Fig. 5a, twist $S_{\boldsymbol{p}}$ can be obtained in terms of limb 1, 2 and 3 separately as

$\boldsymbol{S}_{p}=\sum_{i=1}^{5} \dot{\theta}_{1 i} \boldsymbol{S}_{1 i}$

$\boldsymbol{S}_{p}=\dot{\theta}_{1} \boldsymbol{S}_{a}+\dot{\theta}_{2} \boldsymbol{S}_{b}+\sum_{i=1}^{5} \dot{\theta}_{2 i} \boldsymbol{S}_{2 i}$,

$\boldsymbol{S}_{p}=\dot{\theta}_{4} \boldsymbol{S}_{d}+\dot{\theta}_{5} \boldsymbol{S}_{e}+\sum_{i=1}^{5} \dot{\theta}_{3 i} \boldsymbol{S}_{3 i}$.

Substituting Eqs. (56) and (57) into Eqs. (61) and (62) respectively leads to,

$\boldsymbol{S}_{p}=\dot{\theta}_{1}\left(\boldsymbol{S}_{a}-m_{1} \boldsymbol{S}_{b}\right)+m_{2} \dot{\theta}_{5} \boldsymbol{S}_{b}+\sum_{i=1}^{5} \dot{\theta}_{2 i} \boldsymbol{S}_{2 i}$,

$\boldsymbol{S}_{p}=m_{3} \dot{\theta}_{1} \boldsymbol{S}_{d}+\dot{\theta}_{5}\left(\boldsymbol{S}_{e}-m_{4} \boldsymbol{S}_{d}\right)+\sum_{i=1}^{5} \dot{\theta}_{2 i} \boldsymbol{S}_{2 i}$

where

$$
\begin{aligned}
& m_{1}=\frac{\boldsymbol{P}_{\mathrm{D}} \cdot\left(\boldsymbol{P}_{\mathrm{A}} \times \boldsymbol{P}_{\mathrm{C}}\right)}{\boldsymbol{P}_{\mathrm{D}} \cdot\left(\boldsymbol{P}_{\mathrm{B}} \times \boldsymbol{P}_{\mathrm{C}}\right)}, m_{2}=\frac{\boldsymbol{P}_{\mathrm{D}} \cdot\left(\boldsymbol{P}_{\mathrm{E}} \times \boldsymbol{P}_{\mathrm{C}}\right)}{\boldsymbol{P}_{\mathrm{D}} \cdot\left(\boldsymbol{P}_{\mathrm{B}} \times \boldsymbol{P}_{\mathrm{C}}\right)}, \\
& m_{3}=\frac{\boldsymbol{P}_{\mathrm{B}} \cdot\left(\boldsymbol{P}_{\mathrm{A}} \times \boldsymbol{P}_{\mathrm{C}}\right)}{\boldsymbol{P}_{\mathrm{B}} \cdot\left(\boldsymbol{P}_{\mathrm{D}} \times \boldsymbol{P}_{\mathrm{C}}\right)} \text { and } m_{4}=\frac{\boldsymbol{P}_{\mathrm{B}} \cdot\left(\boldsymbol{P}_{\mathrm{E}} \times \boldsymbol{P}_{\mathrm{C}}\right)}{\boldsymbol{P}_{\mathrm{B}} \cdot\left(\boldsymbol{P}_{\mathrm{D}} \times \boldsymbol{P}_{\mathrm{C}}\right)} .
\end{aligned}
$$

According to [34], we know that the revolute-spherical screws dyad locate in a four-dimensional vector space. So the reciprocal screws form a two-system with zero pitch. Let $\boldsymbol{S}_{i 1}^{r}$ and $\boldsymbol{S}_{i 2}^{r} \quad(i=1,2$ and 3) denote a twosystem of screws that is reciprocal to the four-system of screws $S_{i 2}$ to $S_{i 5}(i=1,2$ and 3) of the $i$ th limb. Performing the reciprocal product of both sides of Eqs. (60), (63) and (64) with reciprocal screw $\boldsymbol{S}_{i 1}^{r}$ and $\boldsymbol{S}_{i 2}^{r}$ gives six linear equations, which can be expressed in matrix form as,

$\mathbf{J}_{q}^{\mathrm{T}} \Delta \boldsymbol{S}_{p}=\mathbf{J}_{\theta} \dot{\boldsymbol{\theta}}_{a}$,

where

$$
\begin{gathered}
\dot{\boldsymbol{\theta}}_{a}=\left[\dot{\theta}_{1}, \dot{\theta}_{5}, \dot{\theta}_{11}, \dot{\theta}_{21}, \dot{\theta}_{31}\right]^{\mathrm{T}} \\
J_{q}^{\mathrm{T}}=\left[\begin{array}{c}
S_{11}^{r T} \\
S_{12}^{r T} \\
\vdots \\
S_{32}^{r T}
\end{array}\right], J_{\theta}=\left[\begin{array}{c}
J_{\theta 1} \\
J_{\theta 2} \\
J_{\theta 3}
\end{array}\right]
\end{gathered}
$$




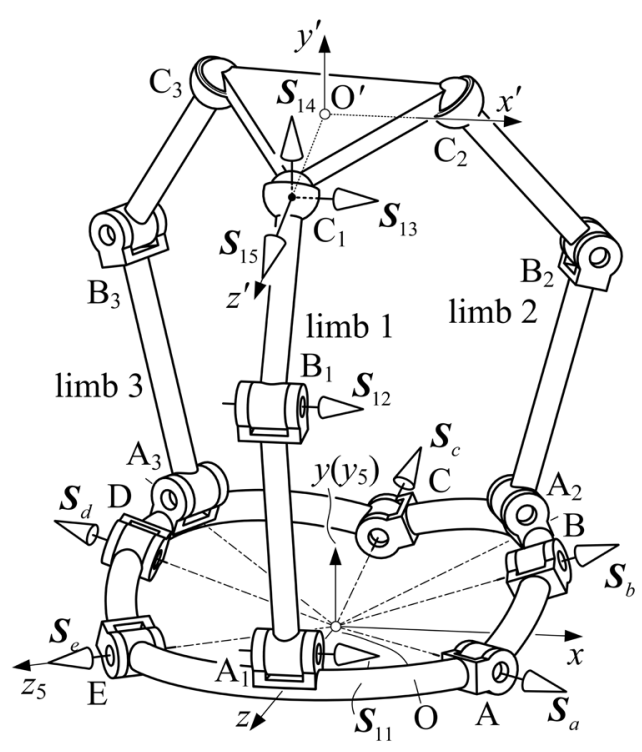

(a)

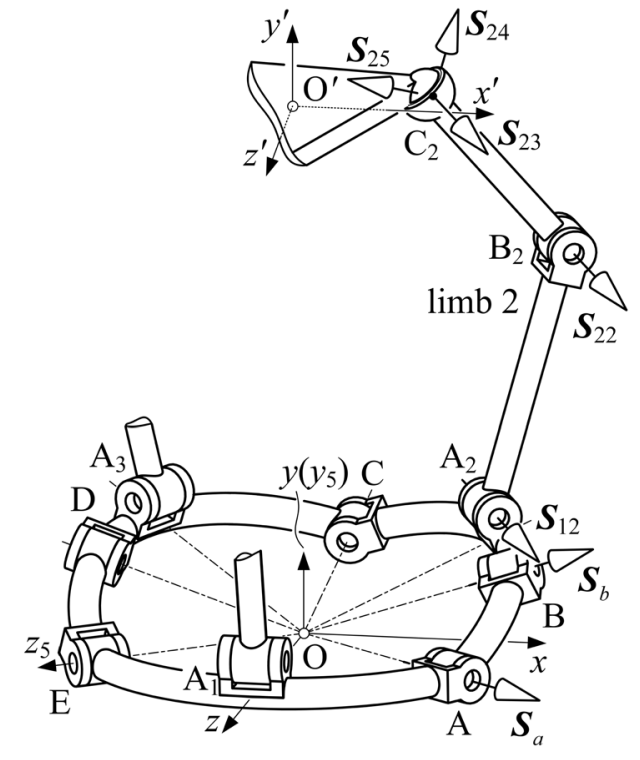

(b)

Fig. 5 Motion screw of the spherical-base integrated parallel mechanism

and $\Delta$ denotes the reciprocal operator expressed as $\Delta=\left[\begin{array}{ll}\mathbf{0} & \mathbf{I} \\ \mathbf{I} & \mathbf{0}\end{array}\right]$.

The term $\mathbf{J}_{\theta i}$ is derived in detail in the Appendix 2, and $\mathbf{J}_{q}^{\mathrm{T}}$, in general, is a $6 \times 6$ nonsingular matrix. Thus, multiplying both sides of Eq. (65) by the inverse of $\mathbf{J}_{q}^{\mathrm{T}}$ gives the twist of the moving platform as,

$\Delta \boldsymbol{S}_{p}=\left[\mathbf{J}_{q}^{\mathrm{T}}\right]^{-1} \mathbf{J}_{\theta} \dot{\boldsymbol{\theta}}_{a}$,

where $\Delta \boldsymbol{S}_{p}$ is the twist of the moving platform with interchange of its primary part and secondary part comparing to $S_{p}$. The left-hand side and right-hand side of Eq. (65) gives the power of the platform and the actuated joints respectively, which provides background for dynamic analysis of the proposed parallel mechanism based on the concept of kinetic energy.

\subsection{Velocity of the spherical-base integrated parallel mechanism}

The spherical-base integrated parallel mechanism can be decomposed as three closed-loop mechanisms between any two of three limbs and an additional closed-loop of the five-bar reconfigurable base. The instantaneous motion of each link can be described as its instantaneous twist, and all the links' motion in a closed-loop form a linear combination of all the instantaneous twists within the loop. Let twist $S_{i j}$ denote the instantaneous motion along the jth joint in the $i$ th limb and twist $\boldsymbol{S}_{a}, \boldsymbol{S}_{b}, \boldsymbol{S}_{c}, \boldsymbol{S}_{d}$ and $\boldsymbol{S}_{e}$ denote that along the joints of the reconfigurable base. Define variable $\dot{\theta}_{i j}$ and $\dot{\theta}_{k}$ as the velocity of the $j$ th joint in the $i$ th limb and the velocity of $k$ th joint in the reconfigurable base. Based on the notations introduced above, twists of three closed-loop-mechanisms are expressed as separately as follows.

For the closed-loop composed of limb 1 and 2, shown in Fig. 6a, the closed-loop-twist is

$\dot{\theta}_{1} \boldsymbol{S}_{a}+\dot{\theta}_{2} \boldsymbol{S}_{b}+\sum_{i=1}^{5} \dot{\theta}_{1 i} \boldsymbol{S}_{1 i}-\sum_{i=1}^{5} \dot{\theta}_{2 i} \boldsymbol{S}_{2 i}=\mathbf{0}$.

For limb 2 and 3, the closed-loop-twist is

$\dot{\theta}_{3} \boldsymbol{S}_{c}+\sum_{i=1}^{5} \dot{\theta}_{2 i} \boldsymbol{S}_{2 i}-\sum_{i=1}^{5} \dot{\theta}_{3 i} \boldsymbol{S}_{3 i}=\mathbf{0}$.

And for limb 3 and 1, the closed-loop-twist is 


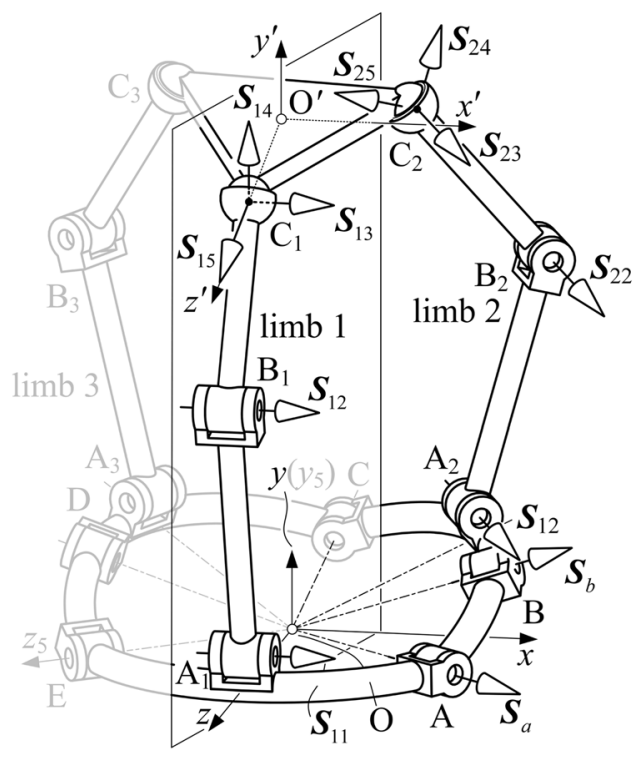

(a)

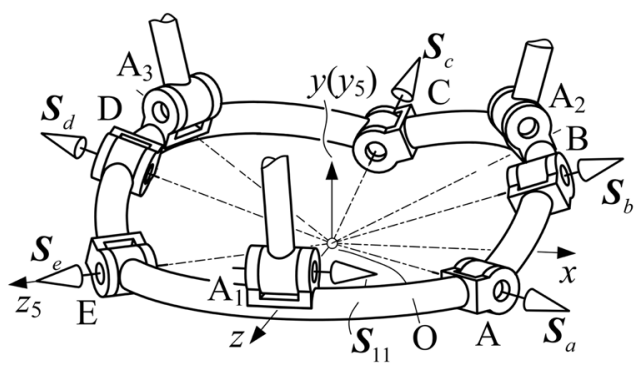

(b)

Fig. 6 Motion screw of closed-loop mechanism decomposed from the parallel mechanism

$\dot{\theta}_{4} \boldsymbol{S}_{d}+\dot{\theta}_{5} \boldsymbol{S}_{e}+\sum_{i=1}^{5} \dot{\theta}_{3 i} \boldsymbol{S}_{3 i}-\sum_{i=1}^{5} \dot{\theta}_{1 i} \boldsymbol{S}_{1 i}=\mathbf{0}$

Further, the closed-loop-twist of the reconfigurable base, shown in Fig. 6(b) is

$\dot{\theta}_{1} \boldsymbol{S}_{a}+\dot{\theta}_{2} \boldsymbol{S}_{b}+\dot{\theta}_{3} \boldsymbol{S}_{c}+\dot{\theta}_{4} \boldsymbol{S}_{d}+\dot{\theta}_{5} \boldsymbol{S}_{e}=\mathbf{0}$.

In each decomposed closed-loop-mechanism, only part of all the joints are active, which can be separated from the rest passive joints in the twists given in Eqs. (67) throughout (70) as,

$\dot{\theta}_{1} \boldsymbol{S}_{a}+\dot{\theta}_{11} \boldsymbol{S}_{11}-\dot{\theta}_{21} \boldsymbol{S}_{21}=-\dot{\theta}_{2} \boldsymbol{S}_{b}-\sum_{i=2}^{5} \dot{\theta}_{1 i} \boldsymbol{S}_{1 i}+\sum_{i=2}^{5} \dot{\theta}_{2 i} \boldsymbol{S}_{2 i}$

$\dot{\theta}_{21} \boldsymbol{S}_{21}-\dot{\theta}_{31} \boldsymbol{S}_{31}=-\dot{\theta}_{3} \boldsymbol{S}_{c}-\sum_{i=2}^{5} \dot{\theta}_{2 i} \boldsymbol{S}_{2 i}+\sum_{i=2}^{5} \dot{\theta}_{3 i} \boldsymbol{S}_{3 i}$,

$$
\begin{aligned}
& \dot{\theta}_{5} \boldsymbol{S}_{e}+\dot{\theta}_{31} \boldsymbol{S}_{31}-\dot{\theta}_{11} \boldsymbol{S}_{11}=-\dot{\theta}_{4} \boldsymbol{S}_{d}-\sum_{i=2}^{5} \dot{\theta}_{3 i} \boldsymbol{S}_{3 i} \\
& +\sum_{i=2}^{5} \dot{\theta}_{1 i} S_{1 i}
\end{aligned}
$$

The Eqs. (71)-(74) can be expressed in a matrix form that indicates the relationship between velocity of the active joints and that passive joints as,

$\mathbf{J}_{a} \dot{\boldsymbol{\theta}}_{a}=\mathbf{J}_{p} \dot{\boldsymbol{\theta}}_{p}$,

where $\dot{\boldsymbol{\theta}}_{a}$ and $\dot{\boldsymbol{\theta}}_{p}$ denote the velocity vectors of active joints and passive joints as, $\dot{\boldsymbol{\theta}}_{P}=\left[\dot{\theta}_{2}, \dot{\theta}_{3}, \dot{\theta}_{4}, \dot{\theta}_{12}\right.$, $\left.\dot{\theta}_{13}, \dot{\theta}_{14}, \dot{\theta}_{15}, \ldots, \dot{\theta}_{35}\right]^{\mathrm{T}}, \quad \mathbf{J}_{a}$ and $\mathbf{J}_{p}$ denote the active-Jacobian matrix and passive-Jacobian matrix respectively as

$$
\mathbf{J}_{a}=\left[\begin{array}{cc:cccc}
\boldsymbol{S}_{1} & \boldsymbol{0} & \boldsymbol{S}_{11} & -\boldsymbol{S}_{21} & \boldsymbol{0} \\
\boldsymbol{0} & \boldsymbol{0} & \boldsymbol{0} & \boldsymbol{S}_{21} & -\boldsymbol{S}_{31} \\
\boldsymbol{0} & \boldsymbol{S}_{5} & -\boldsymbol{S}_{11} & \boldsymbol{0} & \boldsymbol{S}_{31} \\
\boldsymbol{S}_{1} & \boldsymbol{S}_{5} & \boldsymbol{0} & \boldsymbol{0} & \boldsymbol{0}
\end{array}\right] \text { and } \mathbf{J}_{p}=\left[\begin{array}{ccc:ccc}
-\boldsymbol{S}_{2} & \boldsymbol{0} & \boldsymbol{0} & -\mathbf{J}_{p 1} & \mathbf{J}_{p 2} & \boldsymbol{0} \\
\boldsymbol{0} & -\boldsymbol{S}_{3} & \boldsymbol{0} & \boldsymbol{0} & -\mathbf{J}_{p 2} & \mathbf{J}_{p 3} \\
\boldsymbol{0} & \boldsymbol{0} & -\boldsymbol{S}_{4} & \mathbf{J}_{p 1} & \boldsymbol{0} & -\mathbf{J}_{p 3} \\
-\boldsymbol{S}_{2} & -\boldsymbol{S}_{3} & -\boldsymbol{S}_{4} & \boldsymbol{0} & \boldsymbol{0} & \boldsymbol{0}
\end{array}\right]
$$


where $\mathbf{J}_{p i}=\left[\begin{array}{llll}\boldsymbol{S}_{i 2} & \boldsymbol{S}_{i 3} & \boldsymbol{S}_{i 4} & \boldsymbol{S}_{i 5}\end{array}\right], i=1,2$ and 3 .

The above Jacobian matrixes can be used for singularity and dexterity analysis of the proposed integrated parallel mechanism.

\section{Conclusions}

In this paper, a parallel mechanism with a reconfigurable base was for the first time presented based on the manipulation of rigid objects using a metamorphic hand. Structure of the proposed mechanism was presented followed by the geometric constraint analysis. Structure equations of the spherical-base integrated parallel mechanism were then derived and kinematics of the mechanism was investigated, as well as the solutions for forward and inverse kinematics. The screw-based Jacobian was established relationship between active joints and passive joints. This novel spherical-base integrated parallel manipulator has an enlarged workspace compared with traditional parallel mechanisms.

Acknowledgments The authors gratefully acknowledge the support from the European Commission 7th Framework Program SQUIRREL under Grant No. 610532.

Open Access This article is distributed under the terms of the Creative Commons Attribution 4.0 International License (http:// creativecommons.org/licenses/by/4.0/), which permits unrestricted use, distribution, and reproduction in any medium, provided you give appropriate credit to the original author(s) and the source, provide a link to the Creative Commons license, and indicate if changes were made.

\section{Appendix 1}

$$
\begin{aligned}
E_{1}= & e_{1} u_{1}^{2} u_{3}^{2}+e_{2} u_{1}^{2}+e_{3} u_{3}^{2}+e_{6} u_{1} u_{3}+e_{9} \\
E_{2}= & e_{5} u_{1}^{2} u_{3}+e_{7} u_{1} u_{3}^{2}+e_{11} u_{1}+e_{13} u_{3} \\
E_{3}= & e_{4} u_{1}^{2} u_{3}^{2}+e_{8} u_{1}^{2}+e_{10} u_{3}^{2}+e_{12} u_{1} u_{3}+e_{14} \\
F_{1}= & f_{1} u_{1}^{2} u_{3}^{2}+f_{2} u_{3}^{2} u_{1}+f_{4} u_{1}^{2} u_{3}+f_{5} u_{1}^{2}+f_{6} u_{3}^{2}+f_{11} u_{3} \\
& +f_{12} u_{1}+f_{16} \\
F_{2}= & \left(f_{3} u_{1}^{2} u_{3}^{2}+f_{8} u_{1}^{2}+f_{9} u_{3}^{2}+f_{14} u_{1} u_{3}+f_{18}\right) \\
F_{3}= & f_{7} u_{1}^{2} u_{3}^{2}+f_{10} u_{1}^{2} u_{3}+f_{13} u_{3}^{2} u_{1}+f_{15} u_{1}^{2}+f_{17} u_{3}^{2} \\
& +f_{19} u_{3}+f_{20} u_{1}+f_{21}
\end{aligned}
$$

$$
\begin{aligned}
& G_{1}=g_{1} u_{1}^{2}+g_{3} \\
& G_{2}=g_{4} u_{1} \\
& G_{3}=g_{2} u_{1}^{2}+g_{5} \\
& e_{1}=s \delta_{3} c \varphi_{4}-c \delta_{3} s \varphi_{4}-x_{C_{3}^{\prime}} \\
& e_{2}=-s \delta_{3} c \varphi_{4}+c \delta_{3} s \varphi_{4}-x_{C_{3}^{\prime}} \\
& e_{3}=-s \delta_{3} c \varphi_{4}+c \delta_{3} s \varphi_{4}-x_{C_{3}^{\prime}} \\
& e_{4}=-s \delta_{3} c \varphi_{4}-c \delta_{3} s \varphi_{4}-x_{C_{3}^{\prime}} \\
& e_{5}=-4 s \delta_{3} \\
& e_{6}=-2 \\
& e_{7}=-4 c \varphi_{4} \\
& e_{8}=s \delta_{3} c \varphi_{4}+c \delta_{3} s \varphi_{4}-x_{C_{3}^{\prime}} \\
& e_{9}=s \delta_{3} c \varphi_{4}-c \delta_{3} s \varphi_{4}-x_{C_{3}^{\prime}} \\
& e_{10}=s \delta_{3} c \varphi_{4}+c \delta_{3} s \varphi_{4}-x_{C_{3}^{\prime}} \\
& e_{11}=4 c \varphi_{4} \\
& e_{12}=4 \\
& e_{13}=4 s \delta_{3} \\
& e_{14}=-s \delta_{3} c \varphi_{4}-c \delta_{3} s \varphi_{4}-x_{C_{3}^{\prime}} \\
& f_{2}=2 \\
& f_{3}=2 s \delta_{3} \\
& f_{4}=2 s \delta_{3} c \varphi_{4}-2 c \delta_{3} s \varphi_{4} \\
& f_{5}=-y_{C_{3}^{\prime}} \\
& f_{6}=-y_{C_{3}^{\prime}} \\
& f_{7}=-y_{C_{3}^{\prime}} \\
& f_{8}=-2 s \delta_{3} \\
& f_{9}=-2 s \delta_{3} \\
& f_{10}=-2 s \delta_{3} c \varphi_{4}-2 c \delta_{3} s \varphi_{4} \\
& f_{11}=-2 s \delta_{3} c \varphi_{4}+2 c \delta_{3} s \varphi_{4} \\
& f_{12}=-2 \\
& f_{13}=-2 \\
& f_{14}=-8 c \varphi_{4} \\
& f_{15}=-y_{C_{3}^{\prime}} \\
& f_{16}=-y_{C_{3}^{\prime}} \\
& f_{17}=-y_{C_{3}^{\prime}} \\
& f_{18}=2 s \delta_{3} \\
& f_{19}=2 s \delta_{3} c \varphi_{4}+2 c \delta_{3} s \varphi_{4} \\
& f_{20}=2 \\
& f_{21}=-y_{C_{3}^{\prime}}
\end{aligned}
$$




$$
\begin{aligned}
& g_{1}=-c \delta_{3} c \varphi_{4}-s \delta_{3} s \varphi_{4}-z_{C_{3}^{\prime}} \\
& g_{2}=-c \delta_{3} c \varphi_{4}+s \delta_{3} s \varphi_{4}-z_{C_{3}^{\prime}} \\
& g_{3}=c \delta_{3} c \varphi_{4}+s \delta_{3} s \varphi_{4}-z_{C_{3}^{\prime}} \\
& g_{4}=4 s \varphi_{4} \\
& g_{5}=c \delta_{3} c \varphi_{4}-s \delta_{3} s \varphi_{4}-z_{C_{3}^{\prime}}
\end{aligned}
$$

\section{Appendix 2}

$$
\begin{aligned}
\mathbf{J}_{\theta 1} & =\left[\begin{array}{lllll}
\mathbf{0} & \mathbf{0} & \boldsymbol{S}_{11}^{r T} \Delta \boldsymbol{S}_{11} & \mathbf{0} & \mathbf{0} \\
\mathbf{0} & \mathbf{0} & \boldsymbol{S}_{12}^{r T} \Delta \boldsymbol{S}_{11} & \mathbf{0} & \mathbf{0}
\end{array}\right] \\
\mathbf{J}_{\theta 2} & =\left[\begin{array}{lllll}
\boldsymbol{S}_{21}^{r T} \Delta\left(\boldsymbol{S}_{a}-a \boldsymbol{S}_{b}\right) & b \boldsymbol{S}_{21}^{r T} \Delta \boldsymbol{S}_{b} & \mathbf{0} & \boldsymbol{S}_{21}^{r T} \Delta \boldsymbol{S}_{21} & \mathbf{0} \\
\boldsymbol{S}_{22}^{r T} \Delta\left(\boldsymbol{S}_{a}-a \boldsymbol{S}_{b}\right) & b \boldsymbol{S}_{22}^{r T} \Delta \boldsymbol{S}_{b} & \mathbf{0} & \boldsymbol{S}_{22}^{r T} \Delta \boldsymbol{S}_{21} & \mathbf{0}
\end{array}\right] \\
\mathbf{J}_{\theta 3} & =\left[\begin{array}{lllll}
c \boldsymbol{S}_{31}^{r T} \Delta \boldsymbol{S}_{d} & b \boldsymbol{S}_{31}^{r T} \Delta\left(\boldsymbol{S}_{e}-d \boldsymbol{S}_{d}\right) & \mathbf{0} & \mathbf{0} & \boldsymbol{S}_{31}^{r T} \Delta \boldsymbol{S}_{31} \\
c \boldsymbol{S}_{32}^{T} \Delta \boldsymbol{S}_{d} & b \boldsymbol{S}_{32}^{r T} \Delta\left(\boldsymbol{S}_{e}-d \boldsymbol{S}_{d}\right) & \mathbf{0} & \mathbf{0} & \boldsymbol{S}_{32}^{r T} \Delta \boldsymbol{S}_{31}
\end{array}\right]
\end{aligned}
$$

\section{References}

1. Monsarrat B, Gosselin CM (2003) Workspace analysis and optimal design of a 3-leg 6-DOF parallel platform mechanism. Robot Autom IEEE Trans 19(6):954-966

2. Gouttefarde M, Merlet JP, Daney D (2006) Determination of the wrench-closure workspace of 6-DOF parallel cabledriven mechanisms. In: Lennarcic J, Roth B (eds) Advances in robot kinematics. Springer, The Netherlands, pp 315-322

3. Dai JS, Huang Z, Lipkin H (2006) Mobility of overconstrained parallel mechanisms. J Mech Des 128(1):220-229

4. Huang T, Tang G, Li S, Li Y, Chetwynd GD, Whitehouse JD (2003) Kinematic calibration of a class of parallel kinematic machines (PKM) with fewer than six degrees of freedom. Sci China Ser E Technol Sci 46(5):515-526

5. Sun T, Song Y, Li Y, Zhang J (2010) Workspace decomposition based dimensional synthesis of a novel hybrid reconfigurable robot. J Mech Robot 2(3):031009

6. Gogu G (2008) Structural synthesis of parallel robots: part 1 - methodology. Springer, Dordrecht

7. Chablat D, Wenger $P$ (2003) Architecture optimization of a 3-DOF translational parallel mechanism for machining applications, the Orthoglide. Robot Autom IEEE Trans 19(3):403-410

8. Zhao TS, Dai JS, Huang Z (2002) Geometric synthesis of spatial parallel manipulators with fewer than six degrees of freedom. Proc Inst Mech Eng Part C J Mech Eng Sci 216(12):1175-1185

9. Zhao TS, Dai JS, Huang Z (2002) Geometric analysis of overconstrained parallel manipulators with three and four degrees of freedom. JSME Int J Ser C 45(3):730-740
10. Kong X, Gosselin CM (2004) Type synthesis of 3-DOF translational parallel manipulators based on screw theory. J Mech Des 126(1):83-92

11. Xu Q, Li Y (2008) An investigation on mobility and stiffness of a 3-DOF translational parallel manipulator via screw theory. Robot Comput Integr Manuf 24(3):402-414

12. Huda S, Takeda Y (2007) Kinematic analysis and synthesis of a 3-URU pure rotational parallel mechanism with respect to singularity and workspace. J Adv Mech Des Syst Manuf 1(1):81-92

13. Vischer P, Clavel R (2000) Argos: a novel 3-DoF parallel wrist mechanism. Int J Robot Res 19(1):5-11

14. Di Gregorio R (2001) A new parallel wrist using only revolute pairs: the 3-RUU wrist. Robotica 19(3):305-309

15. Gan D, Dai JS (2013) Geometry constraint and branch motion evolution of 3-PUP parallel mechanisms with bifurcated motion. Mech Mach Theory 61:168-183

16. Zhang K, Dai JS, Fang Y (2012) Constraint analysis and bifurcated motion of the 3PUP parallel mechanism. Mech Mach Theory 49:256-269

17. Wang J, Gosselin CM (2004) Kinematic analysis and design of kinematically redundant parallel mechanisms. J Mech Des 126(1):109-118

18. Saglia JA, Tsagarakis NG, Dai JS, Caldwell GD (2009) A high performance redundantly actuated parallel mechanism for ankle rehabilitation. Int J Robot Res 28(9):1216-1227

19. Dai JS, Jones JR (1999) Mobility in metamorphic mechanisms of foldable/erectable kinds. J Mech Des 121(3):375382

20. Gan D, Dai JS, Liao Q (2010) Constraint analysis on mobility change of a novel metamorphic parallel mechanism. Mech Mach Theory 45(12):1864-1876

21. Zhang K, Dai JS, Fang Y (2013) Geometric constraint and mobility variation of two $3 \mathrm{SvPSv}$ metamorphic parallel mechanisms. J Mech Des 135(1):011001

22. Wei G, Dai JS (2014) Origami-inspired integrated planarspherical overconstrained mechanisms. J Mech Des 136(5): 051003

23. Wei G, Dai JS (2014) Reconfigurable and deployable platonic mechanisms with a variable revolute joint. In: Lenarčič J, Khatib O (eds) Latest Advances in Robot Kinematics. Springer, Dordrecht, pp 485-495

24. Yi BJ, Na HY, Lee JH, Hong YS, Oh SR, Suh IH, Kim WK (2002) Design of a parallel-type gripper mechanism. Int J Robot Res 21(7):661-676

25. Mohamed MG, Gosselin CM (2005) Design and analysis of kinematically redundant parallel manipulators with configurable platforms. Robot IEEE Trans 21(3):277-287

26. Lambert P (2013) Parallel robots with configurable platforms. Doctoral dissertation, TU Delft, Delft University of Technology

27. Dai JS Robotic hand with palm Section comprising several parts able to move relative to each other, Patent: WO/2005/ 105391, 2005, International PCT: PCT/GB2005/001665, UK patent: GB04 095 48.5, 2004, Europe patent: EP05740 527.6, US Patent process: US 11/587,766, China Patent: CN200580018189.6

28. Dai JS, Wei G, Wang S, Luo H, Li J (2011) An Anthropomorphic hand with reconfigurable palm. China patent: No. 201110026001.X 
29. Wei G, Stephan F, Aminzadeh V, Würdemann H, Walker R, Dai JS, Gogu G (2014) DEXDEB_-application of DEXtrous robotic hands for DEBoning Operation. In: Rohrbein F et al (eds) Gearing up and accelerating cross-fertilization between academic and industrial robotics research in Europe, Springer tracts in advanced robotics, vol 94, pp 217-235

30. Cui L, Dai JS (2011) Posture, workspace, and manipulability of the metamorphic multifingered hand with an articulated palm. J Mech Robot 3(2):021001

31. Wei G, Dai JS, Wang S, Luo H (2011) Kinematic analysis and prototype of a metamorphic anthropomorphic hand with a reconfigurable palm. Int J Humanoid Rob 8(3):459-479

32. Borras J, Dollar AM (2014) Framework comparison between a multifingered hand and a parallel manipulator. In: Thomas F, Gracia AP (eds) Computational kinematics. Springer, Dordrecht, pp 219-227

33. Borràs J, Dollar AM (2013) A parallel robots framework to study precision grasping and dexterous manipulation. In:
Robotics and Automation (ICRA), 2013 IEEE International Conference on pp 1595-1601. IEEE

34. Dai J S (2014) Screw algebra and Lie groups and Lie algebras, Higher education press, Beijing, also Screw algebra and kinematic approaches for mechanisms and robotics. Springer, London

35. Tsai LW (1999) Robot analysis: the mechanics of serial and parallel manipulators. Wiley, New York

36. Merlet JP (2006) Parallel robots. Springer, Dordrecht

37. Innocenti C, Parenti-Castelli V (1990) Direct position analysis of the Stewart platform mechanism. Mech Mach Theory 25(6):611-621

38. Raghavan M (1993) The Stewart platform of general geometry has 40 configurations. J Mech Des 115(2):277282

39. Roth B (1993) Computations in Kinematics. In: J Angeles et al (eds) Computational kinematics. Springer, Dordrecht, pp 3-14 